\title{
"COBRIR NO PUSCH LA DOLOR QUI•M TURMENTA»: FUENTES IMPRESAS Y TRADICIÓN MANUSCRITA DE UNA POESÍA DE AUSIÀS MARCH SOBRE LA MUERTE*
}

\author{
Llúcia Martín Pascual \\ Universitat d'Alacant \\ 1lucia.martin@ua.es
}

La composición Cobrir no pusch la dolor qui·m em turmenta es la 112 del Cancionero de Ausiàs March según la numeración considerada canónica por Amadeu Pagés en su edición de $1912^{1}$. El tema de la composición se centra en una extensa reflexión sobre la muerte, así como ante el hecho inevitable, la necesidad de entender en su esencia la virtud divina. A diferencia de las poesías conocidas como los Cants de mort, el grupo comprendido entre la 92 y $97^{2}$, aquí no hay referencias a una persona desaparecida, ni al proceso

\footnotetext{
* Trabajo realizado gracias al proyecto «Edición sinóptica de las poesías de Ausiàs March a partir de todos sus testimonios manuscritos e impresos en sus contextos literarios» (FFI2014-52380-C2-1-P), financiado por el Ministerio de Economía y competitividad. Fruto de este proyecto es la primera versión de la edición sinóptica. [En línea]. Enlace: $<$ http://www.cervantesvirtual.com/portales/ausias_march/edicio_sinoptica/>.

${ }^{1}$ Ausiàs March, Les obres d'Auzias March [1912], 2 vols., ed. d'Amadeu Pagès, Barcelona, Institut d'Estudis Catalans, 1990. Las ediciones posteriores, Ausiàs March, Poesies, 5 vols., ed. de Pere Bohigas, Barcelona, Barcino, 1952-59 (cito por la reedición corregida de Amadeu Soberanas y Noemí Espinàs, Barcelona, Barcino, 2000) y A. March, Obra completa, 2 vols., ed. de Robert Archer, Barcelona, Barcanova, 1997, mantienen la numeración establecida por Pagès.

${ }^{2}$ Robert Archer, «Els Cants de Mort», en Aproximació a Ausiàs March, Barcelona, Empúries, 1996, pp. 48-73; Francesc J. Gómez, «Per una nova lectura amorosa i consolatòria dels «Cants de mort» d'Ausiàs March», en Llengua \& Literatura 19 (2008), pp. 49-85 y del mismo autor, «Unitat i seqüència dels "Cants de Mort" d'Ausiàs March", en La poesía d'Ausiàs March i el seu temps, ed. de Ricard Bellveser, Valencia, Publicacions Alfons el Magnànim, 2010, pp 232-262; Arthur Terry, «Per la mort és oberta la carrera: una lectura d'Ausiàs March, poema 92», en Ausiàs March (1400-1459). Premier poète en langue catalane, ed. de Georges Martin, Marie Claire Zimmermann, Paris, Klincksieck, 2000, pp. 231-242, Dominique de Courcelles es la autora de una traducción al francés: Cants de Mort d'Aisuàs March, Valencia, Institució Alfons el Magnànim, 2011.
} 
doloroso de separación de la persona amada, ni a las dudas sobre el destino del alma, ni tampoco tiene una estructura propia de la elegía o el planto ${ }^{3}$. La reflexión de la poesía 112 refiere conceptos abstractos, el dolor y los males que causa la muerte, la anulación de los bienes personales y la soledad que produce la separación. Además, el temor a lo desconocido provoca el deber de afrontar la muerte con fortaleza de ánimo, pero serán incapaces aquellos que quieren conservar los bienes mundanales frente la hipotética felicidad de los sabios que tienen su voluntad unida a Dios ${ }^{4}$. Pere Bohigas, en su edición de las poesías de $\mathrm{March}^{5}$ explica sumariamente el contenido de la composición:

Sobre la mort. El poema comença descrivint la seva obra (vv. 1-90); tot seguit parla dels sentiments que la mort desperta en els homes. Amb aquest motiu el poeta considera diversitat de casos, els quals en línies generals poden ésser objecte de la triple divisió que ell fa de la vida humana: d'acord amb l'esperit, amb la carn o amb el compost psicofísic. Fa particular esment dels qui cerquen la mort voluntària, com feren alguns antics moguts pel desig de glòria, $\mathrm{o}$ confíen que poden sobreposar-se a la por de la mort amb la fortalesa d'ànim. Estretament lligat amb aquests sentiments hi ha el fet dels mals i dels béns que la Mort porta als homes. Totes aquestes coses són analitzades detingudament en el poema (vv. 91-300). La conclusió de March és la següent: només triomfen sobre la Mort els qui creuen que el món i els seus béns -els béns que perdem quan ens morim- són via i no pas fi. L'home no ateny una perfecta coneixença del seu fi amb les virtus morals, que els antics practicaven; només $\mathrm{amb}$ les virtuts divines pot arribar a conèixer el fi superior per al qual ha estat creat, i això li dona l'única arma eficaç per vèncer la Mort (vv. 301-420). El poema acaba invocant la Mare de Déu (420-422).

La composición está formada por 422 versos, lo que la convierte en la segunda más extensa del Cancionero de Ausiàs March después de la poesía 106, otra exhaustiva disertación sobre la virtud de 488 versos. Como es habitual en los poemas de contenido moral March usa la décima, una estrofa de 10 versos con una estructura rítmica propia de la octava: ABBACDDC a la que se añaden dos versos más que riman entre sí EE. En el caso de la poesía que nos ocupa las décimas mantienen cada una rima singular que además se

\footnotetext{
${ }^{3}$ Robert Archer, art. cit., 50-59, expresa la originalidad del ciclo de los Cants de Mort en relación con el género del planctus y los plahns occitanos, precedentes de la poesía de March, así como su vinculación con la tradición consolatoria.

${ }^{4}$ Una interpetación exhaustiva de esta poesía en Francesc J. Gómez, «La meditatio mortis d’Ausiàs March: lectura del cant CXII», comunicación presentada al Congreso El pensament d'Ausiàs March, Barcelona, 10-11 diciembre de 2018.

${ }^{5}$ Bohigas, ed. cit., pp. 405-406.
} 
produce con la combinación de palabras paroxítonas, lo que añade una mayor complejidad al esquema poético, cuando lo habitual es encontrarnos rimas masculinas. La rima femenina de la composición contiene diferentes vocablos procedentes de la derivación: formas verbales como solen, tremolen, algunos homónimos pensa (verbo), pensa (sustantivo), pero también rimas difíciles: nombre-assombre, poble-noble. El esquema es el siguiente: a10' b10' b10' a10' c10' d10' d10' c10' e10' e10'.

La conservación de la poesía 112 en los diferentes testimonios manuscritos e impresos de la obra de March presenta algunas singularidades ${ }^{6}$. El manuscrito que Pagès consideró como base de su edición crítica, el cancionero $F$ (Biblioteca Histórica de la Universidad de Salamanca 2244) ${ }^{7}$, no la contiene, como tampoco otro de los testimonios más completos del siglo XV, el manuscrito $N$ (Biblioteca de la Hispanic Society de Nueva York 2281) ${ }^{8}$, ni los Cancioneros que contienen la obra fragmentaria $(A I L M)^{9}$, todos estos con datación de finales del siglo XV o principios del XVI y, por lo tanto los más antiguos. En cambio, en dos Cancioneros significativos $H$ y $G^{3}$ la poesía se conserva incompleta en una sección diferenciada del resto de las composiciones marquianas. A partir de los manuscritos copiados por Pere de Vilasaló por encargo del Almirante de Nápoles Ferrando Folch de Cardona en 1541 y 1542, la poesía aparece regularmente en los testimonios manuscritos e impresos posteriores. La dedicación de Cardona a la poesía marquiana a mediados del siglo XVI configurará la posterior tradición textual de la obra del poeta

${ }^{6}$ La relación de testimonios manuscritos e impresos se puede consultar en la biblioteca de autor de Ausiàs March del portal Lluís Vives. [En línea]. Enlace: <http://www.cervantesvirtual.com/portales/ausias_march/bibliografia/> [Consulta: 23/04/2020].

${ }^{7}$ Manuscrito de finales del siglo Xv o inicios del XVI que contiene además de un volumen importante de las poesías de Ausiàs March, el Laberinto de Fortuna de Juan de Mena. Completan el documento unas breves poesías de Pere March, Jordi Centelles y Mosén Lluís Pardo.

${ }^{8}$ Este manuscrito solo pudo ser consultado muy parcialmente por Amadeu Pagés para su edición de 1912. Robert Archer realizó un estudio exhaustivo del documento: Robert Archer, «El manuscrit N d'Ausiàs March a la Hispanic Society of America», en Llengua \& Literatura, 4 (1990), pp. 359-422, para incorporar las variantes a su edición de 1996. La posible vinculación entre $F$ y $N$ no puede ser del todo demostrable, Llúcia Martín, «Lecturas divergentes y correcciones de copistas en los manuscritos $F$ y $N$ de las poesías de Ausiàs March», El texto infinito. Tradición y reescritura en la Edad Media y el Renacimiento, ed. de Cesc Esteve, Salamanca, SEMyR, Publicaciones de la Universidad de Salamanca, 2014, pp. 731-747.

${ }^{9}$ Para el estudio de la importancia de estos manuscritos misceláneos en la transmisión de las poesías de March, véase Francesc J. Gómez, «Una antología marquiana a la Barcelona del tercer quart del segle XV», en Revista Valenciana de Filologia 1 (2017), pp. 61-94. Del mismo autor, «La tradició textual d'Ausiàs March en els manuscrits ALM: aportació a un nou stemma codicum», en Llengua \& Literatura, 29 (2019), pp. 59-99. 
valenciano y la formación de un cancionero según el modelo renacentista ${ }^{10}$, del que derivaran las posteriores ediciones impresas del siglo XVI.

En el siguiente cuadro observamos la poesía 112 en todos los testimonios manuscritos e impresos que la contienen, no siempre completa, con algunas de sus características para el posterior análisis:

\begin{tabular}{|c|c|c|c|c|}
\hline TESTIMONIO & FOLIOS & $\begin{array}{l}\text { VERSOS QUE } \\
\text { CONTIENE }\end{array}$ & $\begin{array}{c}\text { VERSOS } \\
\text { QUE FALTAN }\end{array}$ & RÚBRICA \\
\hline$H($ siglo $\mathrm{XV})$ & $295 \mathrm{ra}-297 \mathrm{vb}$ & $1-360$ & $361-422$ & no \\
\hline $\begin{array}{l}G^{3}(1 \mathrm{r} \text { mitad } \\
\text { siglo XVI) }\end{array}$ & $92-98$ & $\begin{array}{l}1-150 \\
161-422 \\
\end{array}$ & $151-160$ (1 estrofa) & no \\
\hline$B(1541)$ & 139r-146r & $\begin{array}{l}1-20 \\
41-422\end{array}$ & $21-40$ ( 2 estrofas $)$ & Altres e son de la mort ${ }^{11}$ \\
\hline$D(1542)$ & $152 \mathrm{v}-162 \mathrm{v}$ & completa & & De la mort \\
\hline$b(1543)$ & $156 r-166 r$ & $1-420$ & Tornada (420-422) & no \\
\hline$c(1545)$ & $159 v-166 v$ & $\begin{array}{l}1-170 \\
191-420\end{array}$ & $\begin{array}{l}171-190 \text { ( } 2 \text { estrofas }) \\
\text { y tornada }\end{array}$ & no \\
\hline$C(1546-47)$ & $215 v-224 r$ & $\begin{array}{l}1-170 \\
191-420\end{array}$ & $\begin{array}{l}171-190 \text { (2 estrofas) } \\
\text { y tornada }\end{array}$ & no \\
\hline$E(1546)$ & $25 \mathrm{r}-31 \mathrm{v}$ & completa & & Mossen Ausiàs March \\
\hline$d(1555)$ & $205 r-215 r$ & completa & & no \\
\hline$e(1560)$ & $198 \mathrm{r}-207 \mathrm{r}$ & completa & & no \\
\hline
\end{tabular}

En el manuscrito $H$, considerado uno de los testimonios más antiguos que conserva de forma fragmentaria la obra de Ausiàs March ${ }^{12}$, la composición ocupa una sección diferenciada del resto del cancionero. Conocido como el Cancionero de Zaragoza, este importante documento misceláneo realiza un recorrido por la poesía del siglo XV con autores más o menos contemporáneos de March como Jordi de Sant Jordi, Lluís de Requesens, Martí Garcia, Lluís

\footnotetext{
${ }^{10}$ La importante labor de Ferrando Folch de Cardona en la conservación y difusión de las poesías de March es analizada detenidamente en el estudio de Albert Lloret, Printing Ausias March. Material culture and Renaissance Poetics, Madrid, Centro para la Edición de los Clásicos Españoles, 2013, especialmente pp. 101-128. Sus ideas renacentistas se materializaron en la creación de una academia en la que participó, entro otros Juan Boscán. Su labor erudita y de mecenazgo, así como su interés por la poesías de Ausiás March le llevó a patrocinar dos copias de su cancionero, los manuscritos $B$ y $K$. Posiblemente también patrocinó la copia del manuscrito $D$, supuesto original de imprenta de la edición de $1543(b)$, impresa a Barcelona en casa de Carles Amorós.

${ }^{11}$ Una rúbrica distintiva de las pocas que contiene este Cancionero.

${ }^{12}$ Jaume Torró, «El Cançoner de Saragossa», en Translatar i transferir. La transmissió dels textos $i$ el saber (1200-1500), ed. de Anna Alberni, Lola Badia y Lluís Cabré, Santa Coloma de Queralt, Obrador Edèndum-Publicacions URV, 2009, pp. 379-423.
} 
de Vilarasa, todos ellos vinculados a la corte de Alfonso el Magnánimo ${ }^{13}$. Otro poeta vinculado con March y presente en este cancionero es Pere de Torroella ${ }^{14}$ y resulta significativo que la obra de ambos se conserve conjunta tanto en $H$ como en $N$.

Retomando la estructura del Cancionero de Zaragoza, las poesías de Ausiàs March se reúnen en 3 secciones:

-Primera sección, ff. 5r-86v poesías amorosas y Cants de Mort, algunas fragmentarias por pérdida de folios.

-Segunda sección, ff. 243r-270v algunas de las poesías consideradas morales, entre otras la 105 -Cant Espiritual- la 106 y la 107.

-Tercera sección: 295r-297v, poesía 112. En esta tercera sección, la poesía 112 está copiada posiblemente por una mano diferente a doble columna, lo que la diferencia también del resto de las composiciones. Permite deducir que se añadió con posterioridad al resto de composiciones del manuscrito ${ }^{15}$. La poesía no está completa ya que solo se han copiado los 360 primeros versos.

El manuscrito $G$ (Biblioteca de la Universidad de Valencia 225) es un documento facticio que contiene mayoritariamente la obra de Ausiàs March, copiada por varias manos y en bloques cronológicamente apartados, tal como ha demostrado Josep Lluís Martos en diferentes trabajos ${ }^{16}$. Como resumen al tratamiento de las secciones de $G$, de acuerdo con los estudios exhaustivos de Martos, Beltran ${ }^{17}$ propone la siguiente distribución:

${ }^{13}$ Autores estudiados en la monografía y edición, Sis poetes de la Cort d'Anfons el Magnànim, ed. de Jaume Torró, Barcelona, Barcino («Els Nostres Clàssics»), 2009.

${ }^{14}$ Pere Torroella, Obra completa, 2 vols., ed. crítica de Francisco Rodríguez Risquete, Barcelona, Barcino («Els Nostres Clàsics»), 2011. March y Torroella también comparten espacio en los Cancioneros AHI ( $I$ es descriptus de A), $L$ y $M$.

${ }^{15}$ Torró, art. cit., considera que la copia de la poesía 112 es tardía, incluida en un cuaderno que posiblemente se añadió al cancionero primitivo con la intención de copiar más textos, entre los que se encuentra esta poesía de March, algunas anónimas y una declaración del duque Joan de Anjou sobre la interrupción de la batalla a ultranza entre Pedro de sant Esteve y Joan Peyró en 1468.

${ }^{16}$ Josep. L. Martos, «Cuadernos y génesis del Cancionero O1 de Ausiàs March (Biblioteca Universitaria de Valencia Ms. 210)», en Actas del II Congreso Internacional «Cancionero de Baena», ed. de J. L. Serrano Reyes, Baena, Ayuntamiento de Baena, 2003, pp. 129-142; del mismo autor, "La gènesi del cançoner $G$ d'Ausiàs March. Les mans dels copistes de $\mathrm{G}^{2} \mathrm{i} \mathrm{G}^{4}$ », en Actas del XIII Congreso de la Asociación Hispánica de Literatura Medieval. In memoriam Alan Deyermond, II, ed. de José Manuel Fradejas Rueda et al., Valladolid, Universidad de Valladolid, 2010, pp. 1349-1359 y también de Josep Lluís Martos, «La duplicació de poemes en el cançoner G d'Ausiàs March i el copista G2b», en Cancionero General, 7 (2009), pp. 35-69.

${ }^{17}$ Vicenç Beltran, Poesia, escriptura i societat: els camins de March, Castelló-Barcelona, Fundació Germà Colón Doménech-Publicacions de l'Abadia de Montserrat, 2006, p. 64. 
G1 más antigua, finales del siglo Xv que, a su vez tiene dos partes

G1a: ff. 23r-91v

Glb: ff. $100 \mathrm{r}-124 \mathrm{v}$

El resto de secciones, copiadas en la primera mitad del siglo XVI

G2: ff. $129 \mathrm{r}-169 \mathrm{v}$

$G^{3}$ : ff. $92 \mathrm{r}-98 \mathrm{v}$

G4: ff. $1-21 \mathrm{r}$

El caso de $G^{3}$ se corresponde a un único cuaderno que constituye una sección diferenciada del resto del manuscrito facticio. Aunque se necesitaría un análisis exhaustivo de variantes, las versiones $H$ y $G^{3}$, en una primera lectura no parecen tener relación de dependencia ${ }^{18}$.

La inclusión de la poesía 112 en estos dos testimonios y las secciones en que se divide el manuscrito $H$, parece indicar que la transmisión más antigua de las poesías de March se produce por dos caminos, el primero el de las poesías propiamente amorosas y el segundo el de otro tipo de composiciones más extensas de carácter moral. Entre las amorosas encontraríamos los seis Cants de Mort, que aún su carácter elegíaco, reflexionan entorno a la idea del amor más allá de la muerte. En este sentido cabe destacar que otros testimonios manuscritos fragmentarios de la poesía de March no contengan las poesías morales -sí las de muerte como en $A / I-$ ni tampoco que este conjunto moral se encuentre completo en los cancioneros más extensos y antiguos como $F$ o $N^{19}$.

La constitución de un verdadero Cancionero de autor de la obra de March se produce en el siglo xvi con la copia de dos manuscritos $B$ y $K$, el manuscrito $D$ y las posteriores ediciones impresas de 1543 y 1545 . Por su parte, el ms. $C$ es una copia de la edición de 1545 , las variantes y su génesis ya fueron analizadas por Josep Lluís Martos así como su estructura ${ }^{20}$. Finalmente, el manuscrito $E$, presenta una ordenación alfabética completamente diferente

\footnotetext{
${ }^{18}$ Un primer estudio de las variantes se realizó en Llúcia Martín, «Noves propostes de lectura a partir de l'elaboració d'una edició sinóptica de les poesies d'Ausiàs March», comunicación presentada en el XVIII congreso de la Asociación Hispánica de Literatura Medieval, Barcelona, 2-6 de septiembre de 2019, publicación en prensa: "Prenga xascú ço qui Millor li és de mon dit»: creació, recepció i representació de la literatura medieval, San Millán de la Cogolla, Cilengua.

${ }^{19}$ Entre otras composiciones, estos testimonios contienen las poesías 106 y 107, que coinciden con $H$. También coinciden $F H$ en la versión incompleta del Cant Espiritual que, sin embargo no aparece en $N$.

${ }^{20}$ Josep Lluís Martos, "Ausiàs March en Italia: variantes y contextos de un "codex descriptus"», en Revista de poética medieval, 28 (2014), pp. 265-294.
} 
del resto de testimonios ${ }^{21}$, ya que se copió después de las impresiones de 1543 y 1545 con su posible influencia, tal como se indica en el prólogo ${ }^{22}$.

La situación de la poesía 112 en los testimonios impresos presenta las siguientes peculiaridades:

\begin{tabular}{|l|l|}
\hline IMPRESO & SECCIÓN \\
\hline$b$ & Falta tornada, versos 421-422. Sección Obres morals \\
\hline$c / C$ & $\begin{array}{l}\text { Además de la tornada (421-22) faltan los versos 171-190. El resto coin- } \\
\text { cide con } b . \text { Sección Obres morals }\end{array}$ \\
\hline$d$ & $\begin{array}{l}\text { Completa. La ordenación de las estrofas coincide con b/c hasta el verso } \\
\text { 281. Sección Obras de muerte }\end{array}$ \\
\hline$e$ & $\begin{array}{l}\text { Coincide completamente con la estructura de } d, \text { lo que confirma la de- } \\
\text { pendencia de este impreso de } 1560 \text { con } d(1555) \text {. Sección } \text { Obres de mort. }\end{array}$ \\
\hline
\end{tabular}

La estructura de los cancioneros impresos difiere de la de los manuscritos por la agrupación en secciones (Cants d'amor, de mort y morals) y por el proceso de reestructuración a que se vio sometida la poesía de March. Esta división en secciones ya aparece en $1539(a)^{23}$, una antología preparada por Baltasar de Romaní de 46 composiciones con sus correspondientes traducciones al castellano, dirigida al Duque de Calabria y publicada en Valencia por Joan Navarro, otro centro cultural y poético importante ${ }^{24}$ junto con la Barcelona de mediados de siglo XVI.

Observamos detenidamente los grupos de poesías no amorosas que conforman las secciones de los cancioneros impresos:

Edición de 1543 (b). La sección Obres Morals cierra el cancionero y a pesar que no se destaca con una rúbrica distintiva, sabemos que se trata de una determinada parte de las obras poéticas por el encabezamiento que abarca los ff. 135v-172r. Dicha sección de las poesías morales está formada por las poesías siguientes (entre paréntesis el número de la composición según la ordenación de Pagès):

\footnotetext{
${ }^{21}$ Maria Mercè López Casas, «De los impresos al cancionero E de Ausiàs March», en Del impreso al manuscrito en los cancioneros, ed. de Josep Lluís Martos, Alcalá de Henares, Centro de Estudios Cervantinos, 2011, pp. 171-186; y de la misma autora «Lectores humanistas y doble versión: variantes impresas para el cancionero manuscrito E de Ausiàs March» en Variación y testimonio único. La reescritura de la poesia ed. de Josep Lluís Martos, Alacant, Universitat d'Alacant, 2017, pp. 181-196.

${ }^{22} \mathrm{El}$ manuscrito está dedicado a Àngela Carroç de Vilaragut, dama valenciana lectora de March. En el prólogo se da noticia que apra la copia del mansucrito se ha recurrido a las ediciones de Barcelona de 1543 y 1545, así como a documentación referida a la biografía de Ausiàs March.

${ }^{23}$ La edición de 1539 no contiene la poesia 112.

${ }^{24}$ Lloret, op. cit., pp. 57-98 estudia el proceso de edición y traducción de la obra de March en esta antología, con especial atención al cuidado del editor de evitar referencias susceptibles de inmoralidad.
} 
Aquell ateny tot quant atenyer vol (103)

Qui de per si ne per Deu virtuts usa (104)

Lo tot es poch ço per que treballam (106)

La vida's breu e l'art se mostra longa (113)

O quant es foll qui tem lo forçat cas (107)

Cobrir no puc la dolor qui·n turmenta (112)

No·m clam d'algu que un mon mal haja colpa (108)

Puix que sens tu algu a tu no basta $(105)^{25}$.

La misma estructura y conjunto de composiciones se repite en la edición de 1545 , también sin rúbricas distintivas por secciones y solo identificadas como conjunto de Obres morals por el encabezamiento de página, ocupando los ff. $137 \mathrm{v}-172 \mathrm{v}^{26}$. En el manuscrito $C$ encontramos la misma correspondencia, ya que es copia del impreso, pero sin el encabezamiento ni las rúbricas.

Los cancioneros impresos de 1555 (d) y 1560 (e) contienen la poesía 112 en la sección dedicada a las obras de muerte, localizada al final del volumen con la rúbrica Siguense las obras de muerte del ingeniossíssimo y elegantissimo Mossen Ausias March (1555) ${ }^{27}$ que ocupa los ff. 188r-215r, y Seguexense las obras de mort dels ingeniosíssim y elegant poeta mossén Ausiàs March (1560) que ocupa los ff. 181r-207r, también al final de la edición, en la que se ofrece un conjunto de 8 poesías.

Aquelles mas que jamés perdonaren (92).

O quant és foll qui tem lo forçat cas (107).

Qui ser·aquell del món superior (93).

Puix me trob sol en amor, a mi sembla (94).

Que val delit puix no és conegut (95).

Si per null temps creguí ser amador (97).

La gran dolor que lengua no pot dir (96).

Cobrir no puch la dolor qui $\cdot m$ turmenta (112).

${ }^{25}$ Se trata del Cant Espiritual que en algunos testimonios se diferencia de las obras morales, pero en otros no o bien va precedido de la rúbrica Oracio. Vease Rafael Alemany, «L'anomenat 'Cant espiritual' d'Ausiàs March en els testimonis antics de l'obra del poeta», en Revista Valenciana de Filologia, 1 (2017) pp. 13-33, y del mismo autor, «La cántica espiritual de la primera edición de las poesías de Ausiàs March», Avatares y perspectivas del medievalismo ibérico, vol. II, coord. de Isabella Tomassetti, San Millán de la Cogolla, Cilengua, 2019, pp. 999-1014.

${ }^{26}$ Por un error de los impresores la primera poesía del grupo de obras morales, Aquell ateny tot quant atenyer vol aún lleva como encabezamiento «Obres de mort».

${ }^{27}$ Todos los paratexots y rúbricas de este Cancionero son en castellano, posiblemente por el origen de la impresión realizada en Valladolid. 
En resumen, los cancioneros $a, b$ y $c$ contienen la sección de Obras Morales al final del volumen, solo en los dos casos más tardíos $d$ y e, la sección Obres de Mort se ha desplazado al final del cancionero, añadiendo dos composiciones más que no forman parte de esta tradición. Además solo en estos dos últimos cancioneros las obras morales se anteponen a las de muerte. Por otra parte, el impreso $b$ (1543) reproduce el contenido poético de su posible original de imprenta, el manuscrito $D^{28}$, un testimonio que presenta una serie de marcas y anotaciones que parecen indicar el sistema de ordenación para seguir por los impresores.

La poesía de March se ha sometido, pues, a un riguroso estudio y reordenación que aleja manuscritos e impresos y que obedece a la moda de los cancioneros del Renacimiento y en concreto a la difusión del Canzionere de Petrarca. En $b$ y $c$ este proceso de reestructuración deriva aún de una primera disposición alejada de los manuscritos pero con un sentido de cancionero personal y cronológico: obras de amor, de muerte y obras morales. En $d$ y $e$ la obra de March se somete a otro proceso de reordenación que desplaza poesías morales y obras de muerte al final, por no decir de otras composiciones consideradas amorosas que también se incluyen en las morales. Incluso se abre una sección diferente para las poesías «esparsas», es decir aquellas que solo están formadas por una estrofa y que no siempre aparecen en todos los testimonios.

En las dos últimas ediciones impresas, $d$ y $e^{29}$, el grupo de los seis Cants de Mort que habitualmente aparecen como un conjunto cohesionado, presentan la adición de los poemas 112 y 107 . Ambos tratan el tema de la muerte, el 107 es una elegía por la muerte de Mossén Borra ${ }^{30}$ mientras que el poema 112 es de tono introspectivo pero sin un referente concreto, como se ha indicado. Ahora bien, hasta este momento ni una composición ni la otra se relacionaba con los Cants de Mort. Nuestra hipótesis es la de un progresivo desplazamiento de los seis Cants de Mort (92-97) como poesías dedicadas a la muerte de la amada, y por tanto de temática amorosa, a una interpretación moralizante, en la que tienen cabida otro tipo de poesía discursiva sobre la muerte.

${ }^{28}$ Mercè López-Casas, «¿El Cancionero $D$ de Ausiàs March, un original de imprenta?», en Actas del XIII Congreso Internacional de la Asociación Hispánica de Literatura Medieval. In memoriam Alan Deyermond, II, ed. de José Manuel Fradejas Rueda et al., Valladolid, Universidad de Valladolid, 2010, pp. 1181-1200.

${ }^{29}$ Ver el estudio sobre la estructura del cancionero impreso $e$ de Mercé López-Casas, «Claudi Bornat, editor d'Ausiàs March? Estudi material d'un cançoner imprés», en eHumanista/IVITRA 13 (2018), pp. $472-488$.

${ }^{30}$ Seguramente se trate de Antoni Tallander, amigo del poeta y persona muy vinculada a la corte real de Alfonso el Magnànimo, Bohigas, ed. cit., p. 381. 
Los copistas de los cancioneros y también los primeros editores interpretaron estos cantos de muerte como una continuidad de los amorosos, centrados en la desaparición de la persona amada y las dudas que genera la pervivencia del amor. En cambio las ediciones posteriores no observan o no entienden esta interpretación y en el proceso de reordenación añaden poesías sobre el hecho luctuoso que no tienen relación con el dolor por la muerte de la aymia.

A continuación, ofrecemos un análisis de los impresos que contienen la poesía objeto de nuestro estudio con sus peculiaridades, variantes y posible filiación entre testimonios impresos i/o manuscritos. Los ejemplares utilizados para el estudio de la poesía 112 son, principalmente, los que se contienen digitalizados en el espacio dedicado a Ausiàs March en la Biblioteca Virtual Miguel de Cervantes, aunque también se han consultado otros ejemplares como se indicará oportunamente ${ }^{31}$.

A excepción de la editio princeps de 1539 que solo reproduce una antología poética con sus correspondientes traducciones, el resto de ediciones sí que contienen la poesía 112. La tradición impresa de esta poesía comienza, pues, con la edición de 1543, realizada por Carles Amorós en Barcelona, bajo los auspicios de Ferrando Folch de Cardona y seguramente tomando como manuscrito original el texto $D$ (BNE 2985). De esta edición Les obres de mossen avsias march. ab vna declaratio en los marges. de algvns vocables scvrs se conservan, según Biteca ${ }^{32}, 22$ ejemplares, no contiene paratextos ni rúbricas, solo para indicar la tornada, pero como anuncia el título, hay una serie de anotaciones al margen que pretenden traducir o por lo menos anotar algunas palabras ininteligibles o arcaicas. Estas anotaciones desaparecen a partir del f. 102v, por lo que no afectan a la poesía 112, localizada entre los ff. 157 v y 166r. Sin embargo, el ejemplar R-3192 de la Biblioteca Nacional de España contiene una serie de escolios a mano, posiblemente de dos lectores diferentes, que añaden interés al ejemplar por el tipo de explicaciones que les sugiere la lectura de la compleja poesía marquiana ${ }^{33}$. Observamos estas anotaciones que afectan a la nuestra poesía en el cuadro siguiente:

\footnotetext{
${ }^{31}$ [En línea]. Enlace: <http://www.cervantesvirtual.com/portales/ausias_march/> [Consulta: 28/01/2020].

${ }^{32}$ BITECA. Bibliografia de textos catalans antics. [En línea]. Enlace: $<$ https://bancroft.berkeley.edu/ philobiblon/biteca_ca.html> [Consulta: 28/01/2020]. Las características materiales de los diferentes impresos aparecen detalladas en esta base.

${ }^{33}$ Joan Mahiques, «Escolis y versos manuscrits a les edicions d'Ausiàs March», en El (re)descobriment de l'edat moderna. Estudis en homenatge a Eulàlia Duran, 2007, pp. 281-295.
} 
«COBRIR NO PUSCH LA DOLOR QUI·M TURMENTA»: FUENTES IMPRESAS Y TRADICIÓN

\begin{tabular}{|c|c|c|}
\hline VERSO & PALABRA O EXPRESIÓN & ANOTACIÓN AL CASTELLANO \\
\hline 9 & velledat & vejez \\
\hline 69 & ta fort dolor & tu fuerte dolor \\
\hline 73 & pedres & piedras \\
\hline 73 & roques & rocas \\
\hline 127 & bresca & panal de miel \\
\hline 170 & closa & ençerrada \\
\hline 174 & ferea & miedo o verguença desa fiera \\
\hline 180 & alleuja & alibiana \\
\hline 189 & assajen & pruevan $^{34}$ \\
\hline 190 & vajen & vayan \\
\hline 208 & data & la data tras la [..] cion del Juego de tablas \\
\hline 218 & desisca & desista \\
\hline 219 & jutje & Iuez \\
\hline 220 & jutje & Iuzgue \\
\hline 221 & senys & señales \\
\hline 226 & lestes & leydas \\
\hline 227 & gestes & hechos \\
\hline 241 & bateja & se baptiza o toma nombre \\
\hline 253 & costa & debe ser decosta \\
\hline 254 & sesmenta & no se olvjda \\
\hline 255 & senys & sentidos \\
\hline 256 & exces & eçessos endemas \\
\hline 257 & armari & armari, el almario que nes el cuerpo donde estava el Alma \\
\hline 265 & sesdeue & acaesçe o abiene \\
\hline 265 & visquen & biuen \\
\hline 268 & desisquen & desistan \\
\hline 320 & vmple & hinche \\
\hline 323 & promes & buenos y abonados, o prohivido \\
\hline 324 & Raels & raízes \\
\hline 328 & vells & viejos \\
\hline 348 & pobra & pobre \\
\hline 391 & Algun tirant & estrofa señalada con una manícula \\
\hline 399 & ampra & $\begin{array}{l}\text { [a]mpra busca prestado } \\
\text { [a]mprar tomar prestado } \\
\text { [a]mprar deprener o apro[..]barse } \\
\text { [a]mpra ampara o defiende } \\
\text { [a]mpre yo me aprobeche }\end{array}$ \\
\hline 411 & hi & allí o aqui \\
\hline
\end{tabular}

\footnotetext{
${ }^{34}$ Caligrafía diferente de las anteriores notas, podría ser una mano diferente. También en la nota al verso 190.
} 
La edición de 1545 (c), también impresa por Carles Amorós con el título Les Obres del valeros y extrenv caualler vigil y elegantissim poeta Ausias March : Nouament reuistes y estampades posades totes les declarasions dels vocables scurs molt largament en la taula, tampoco contiene paratextos ni los «mots obscurs» al margen sino al final del ejemplar en una especie de glosario $^{35}$. Se considera una reedición de la anterior, incluso la tipografía es semejante y, como tendremos ocasión de comprobar en relación con la poesía 112, se introducen algunas correcciones, aunque también aparecen erratas. Según Biteca se conservan 19 ejemplares además de una copia manuscrita, el manuscrito $C$ en la Biblioteca del Real Monasterio del Escorial (ms. L.iij.26) ${ }^{36}$. La portada contiene un grabado que representa un fénix con la leyenda: Mereix qui feu els obres daquest llibre renom de Foenix home.

La edición $d$, impresa en Valladolid en 1555 por Sebastián Martínez bajo el título Las obras del poeta mossén Ausiàs March, corregidas de los errores que tenían. Sale con ellas el vocabulario de los vocablos en ellas contenido. Dirigidos al illustríssimo señor Gonçalo Fernández de Córdova, duque de Sesa y de Terranova, conde de Cabra, señor de la casa de Vaena, etc., contiene diferentes paratextos, la división en secciones con sus correspondientes rúbricas, la numeración de las poesías (De amor canto primo...) y la indicación del final de cada poesía, algo que no aparecía en las ediciones anteriores y podía dificultar la lectura al no advertir claramente el final de cada composición por la ausencia de rúbricas iniciales. El glosario, ampliado respecto a las ediciones anteriores constituye un auténtico diccionario con unas lecciones de gramática de la lengua «lemosina» a cargo de Juan de Resa ${ }^{37}$. Entre los paratextos de esta edición, encontramos una epístola dirigida al Duque de Sesa, un soneto dedicado a Ausiàs March de Jorge de Montemayor y una breve biografía de Ausiàs March. Según Biteca se conservan 34 ejemplares dispersos en bibliotecas del mundo.

Por último la edición e, impresa en Barcelona en 1560 por Claudi Bornat lleva por título Les Obres del valeros cavaller y elegantissim poeta Ausias March. Ara nouament ab molta dilige[n]cia reuistes y ordenades y de molts

${ }^{35}$ El ejemplar digitalizado en la Biblioteca Virtual Cervantes está incompleto por la falta de los folios finales. Se puede consultar el que se conserva en la Biblioteca Valenciana Signatura: XVI/493.

${ }^{36}$ Este manuscrito también presenta una serie de anotaciones al margen que no tienen relación con los impresos «mots obscurs». Véase Pere Ramirez i Molas, «Un manuscrit inèdit d'Ausiàs March», en Estudis de Llengua i Literatura Catalanes, 2 (=Homenatge a Josep M. Casacuberta, 2), Barcelona, Publicacions de l'Abadia de Montserrat, 1981, pp. 217-240.

${ }^{37}$ Germà Colón, «Ausiàs March interpretat al segle Xvı per Juan de Resa i Jorge de Montemayor», en Ausiàs March, textos i contextos, ed. de Rafael Alemany, Alacant-Barcelona, Institut Interuniversitari de Filologia Valenciana-Publicacions de l'Abadia de Montserrat, 1997, pp. 89-116. 
$c a[n]$ ts aume [n]tades y se caracteriza por una diferente tipografía, sobre todo en las rúbricas. Contiene varios paratextos, como la dedicatoria al Duque de Soma, Ferrando Folch de Cardona (el mismo mecenas de los manuscritos y la edición de 1543), un soneto de Pere Serafí, varias poesías de Francesc Calça, algunas en latín, y se omite la referencia biográfica de la edición de 1555. A pesar de su semejanza con la edición de 1555, ya que cuenta con la misma estructura de las poesías en secciones con sus correspondientes rúbricas, la composición de este impreso parece obedecer a una iniciativa diferente. Se conservan 37 ejemplares de esta edición.

Centrándonos en la poesía 112, observamos su disposición en los diferentes impresos.

1. Estrofas por página. La edición $b$ de $1543^{38}$ contiene unos 20 versos por página aproximadamente en las poesías en octavas, número que varía si se trata de una tornada que necesariamente va encabezada por una rúbrica. Respecto las poesías en décimas, la disposición es de 24 versos por página por regla general, es decir dos estrofas completas más cuatro o hasta seis versos de otra estrofa. Cabe destacar que las décimas van subdividas en 8 versos más 2 y se ha dejado un espacio entre los dos conjuntos $8+$ espacio + dos. Los dos últimos versos van siempre encabezados por letra mayúscula. Esta separación no se produce regularmente en $c$ por lo que la disposición que encontramos es también de unos 24 o 26 versos por página y aparentemente sin ninguna apreciación de orden en el corte de estrofas. En $d$ es habitual la separación de la décima en $8+2$, aunque no en todas las estrofas, de manera que quedan dos décimas por página. Por último, en la edición $e$ sí que encontramos la separación en estrofas en dos conjuntos $8+$ espacio + dos, de manera que hay dos décimas por página aunque no completas y sin orden concreto en el corte.

La edición $b$ no tiene números de folios, aunque hemos otorgado una paginación para facilitar la transcripción. La poesía ocupa parte de los cuadernos $\mathrm{V}$ y X, como podemos observar por las iniciales a pie de página. La edición $c$ de 1545 presenta foliación al margen superior derecho pero también la estructura habitual por cuadernos. La poesía, como en la edición de 1543, ocupa parte de los cuadernos V y X. Las ediciones más modernas de 1555 y 1560 contienen tanto rúbricas de cuadernos como foliación y en este caso la poesía se extiende en parte de los cuadernos CC /DD. La composición 112 ocupa 16 páginas en $c, 18$ en $b$ y 21 en $d$ y $e$, de tamaño menor que las ediciones anteriores. Es habitual encontrar también reclamos, incluso en la edición de 1555

\footnotetext{
${ }^{38}$ Es una impresión de mayores dimensiones que el resto de ediciones, por lo que la disposición no siempre se corresponde en todos los impresos. Ver Biteca para las descripciones materiales.
} 
los hay en todas las páginas. La menor extensión de $c$ se debe a la pérdida de dos estrofas en el impreso.

2. Estrofas omitidas. No encontramos una razón derivada del documento para explicar la pérdida de dos estrofas, vv. 171-190 en $c$, simplemente que el f. 162r termina con una copla completa y por descuido del impresor se omitirían las dos que faltan. En este caso es posible que no se tuviera delante la impresión de 1543 donde las estrofas ocupan diversas páginas y no coinciden los finales de página con el final de estrofa. Nos preguntamos si se trabajaría con otro original. Aun siendo así, las lecciones son muy similares, con algunas erratas en $c$, por lo que dependerían de un mismo testimonio, con toda probabilidad el manuscrito $D$. Una última omisión que cabe destacar es la de la tornada de dos versos de $D$ que no aparece en los impresos $b$ y $c$ pero que $d$ y $e$ recuperan.

3. Disposición y orden del texto de la poesía 112. A excepción de las estrofas omitidas en $c$, la disposición del texto es la misma en $b$ y $c$, en cambio en $d$ y $e$ difiere a partir del verso 281 :

\begin{tabular}{|c|c|c|c|c|}
\hline VERSOS & $\mathrm{b}$ & $\mathrm{c}$ & d & e \\
\hline 281 & $\begin{array}{l}\text { Per delitar / los homens } \\
\text { vida volen }\end{array}$ & $\begin{array}{l}\text { Per delitar / los homens } \\
\text { vida volen }\end{array}$ & $\begin{array}{l}\text { [212r] Tot quant se te / } \\
\text { per qual que sostinença }\end{array}$ & $\begin{array}{l}\text { Tot quant se te / per } \\
\text { qualque sostinença }\end{array}$ \\
\hline 291 & $\begin{array}{l}\text { Tals son, e tants / los } \\
\text { mals que mort atraça }\end{array}$ & $\begin{array}{l}\text { Tals son, e tants / los } \\
\text { mals que mort atraço }\end{array}$ & $\begin{array}{l}\text { Puis que del mon / e } \\
\text { dins si l'hom nos farta }\end{array}$ & $\begin{array}{l}\text { Puix que del mon / e dins } \\
\text { si l'hom nos farta }\end{array}$ \\
\hline 301 & $\begin{array}{l}\text { Qui en virtuts / morals, } \\
\text { ha vida feta }\end{array}$ & $\begin{array}{l}\text { [164v] Qui en virtuts / } \\
\text { morals, ha vida fet }\end{array}$ & $\begin{array}{l}\text { [212v] Quil espirit / } \\
\text { infinit lo vol creure }\end{array}$ & $\begin{array}{l}\text { Quil esperit / infinit lo } \\
\text { vol creure }\end{array}$ \\
\hline 311 & $\begin{array}{l}{[164 r] \text { Menys de ser bo / }} \\
\text { per son saber hmo basta }\end{array}$ & $\begin{array}{l}\text { Menys de ser bo / per } \\
\text { son saber hom basta }\end{array}$ & \begin{tabular}{|l|}
$\begin{array}{l}\text { Deu es tot be / e lo mon } \\
\text { son contrari }\end{array}$ \\
\end{tabular} & $\begin{array}{l}\text { Deu es tot be / e lo mon } \\
\text { son contrari }\end{array}$ \\
\hline 321 & $\begin{array}{l}\text { Primer que leys / fossen } \\
\text { per Deu donades }\end{array}$ & $\begin{array}{l}\text { Primer que leys / fossen } \\
\text { per Deu donades }\end{array}$ & $\begin{array}{l}\text { [213r] Qui no vol be / } \\
\text { forcat es que mal vulla }\end{array}$ & $\begin{array}{l}\text { Qui no vol be / forçat es } \\
\text { que mal vulla, }\end{array}$ \\
\hline 331 & $\begin{array}{l}\text { Tres principals / fins son } \\
\text { d'hon homens obren }\end{array}$ & $\begin{array}{l}\text { Tres principals / fins son } \\
\text { d'hon homens obren }\end{array}$ & $\begin{array}{l}\text { Algun tirant / no feu tan } \\
\text { alta'npresa }\end{array}$ & $\begin{array}{l}\text { Algun tirant / no feu tan } \\
\text { alta'npresa }\end{array}$ \\
\hline 341 & $\begin{array}{l}\text { Tot quant se te / per } \\
\text { qualque sostinença }\end{array}$ & $\begin{array}{l}\text { Tot quant se te / per } \\
\text { qualque sostinença }\end{array}$ & $\begin{array}{l}{[213 v] \text { Per delitar / los }} \\
\text { homens vida volen }\end{array}$ & $\begin{array}{l}\text { Per delitar / los homens } \\
\text { vida volen }\end{array}$ \\
\hline 351 & $\begin{array}{l}\text { Puix que del mon / e } \\
\text { dins si l'hom nos farta }\end{array}$ & $\begin{array}{l}\text { Puix que del mon / e } \\
\text { dins si l'hom nos farta }\end{array}$ & $\begin{array}{l}\text { Tals son e tants / los } \\
\text { mals que Mort atraça }\end{array}$ & $\begin{array}{l}\text { Tals son e tants / los mals } \\
\text { que Mort atraça }\end{array}$ \\
\hline 361 & $\begin{array}{l}\text { [165r] Quil esperit / } \\
\text { infinit lo vol creure }\end{array}$ & $\begin{array}{l}\text { Quil esperit / infinit lo } \\
\text { vol creure }\end{array}$ & $\begin{array}{l}\text { [214r] Qui en virtuts / } \\
\text { morals, ha vida feta, }\end{array}$ & $\begin{array}{l}\text { Qui en virtuts / morals, } \\
\text { ha vida feta, }\end{array}$ \\
\hline 371 & $\begin{array}{l}\text { Deu es tot be / e lo mon } \\
\text { son contrari }\end{array}$ & $\begin{array}{l}\text { Deu es tot be / e lo mon } \\
\text { son contrari }\end{array}$ & $\begin{array}{l}\text { Menys de ser bo / per } \\
\text { son saber l'hom basta }\end{array}$ & $\begin{array}{l}\text { [206r] Menys de ser bo / } \\
\text { per son saber l'hom basta }\end{array}$ \\
\hline 381 & $\begin{array}{l}\text { Qui no vol be / foarçat } \\
\text { es que mal vulla }\end{array}$ & $\begin{array}{l}\text { Qui no vol be /forçat es } \\
\text { que mal vuulla }\end{array}$ & $\begin{array}{l}\text { [214v] Primer que leys / } \\
\text { fosen per Deu donádes }\end{array}$ & $\begin{array}{l}\text { Primer que leys / fossen } \\
\text { per Deu donádes }\end{array}$ \\
\hline 391 & $\begin{array}{l}\text { Algun tirant / no feu tan } \\
\text { alta'mpresa }\end{array}$ & $\begin{array}{l}\text { Algu tirant / no feu tan } \\
\text { alta'mpresa }\end{array}$ & $\begin{array}{l}\text { Tres prinçípals / fins,son } \\
\text { de hon homens obren }\end{array}$ & $\begin{array}{l}\text { Tres principals / fins, son } \\
\text { d'hon homens obren }\end{array}$ \\
\hline 401 & $\begin{array}{l}\text { Mort, lo mon tol / y al } \\
\text { conquistant conquista }\end{array}$ & $\begin{array}{l}\text { Mort lo mon tol / y al } \\
\text { conquistant conquista }\end{array}$ & $\begin{array}{l}\text { [215r] Mort lo mon tol/ } \\
\text { yal conquistant conquista }\end{array}$ & $\begin{array}{l}\text { Mort lo mon tol / y al } \\
\text { conquistant conquista }\end{array}$ \\
\hline
\end{tabular}


«COBRIR NO PUSCH LA DOLOR QUI·M TURMENTA»: FUENTES IMPRESAS Y TRADICIÓN

\begin{tabular}{|l|l|l|l|l|}
\hline 411 & $\begin{array}{l}166 \mathrm{r}] \mathrm{Fe}, \text { caritat / y } \\
\text { esperançans hi porten }\end{array}$ & $\begin{array}{l}{[166 \mathrm{v}] \mathrm{Fe}, \text { caritat / y }} \\
\text { esperançans hi porten }\end{array}$ & $\begin{array}{l}\text { Fe,charitat / y } \\
\text { esperançan's hi porten }\end{array}$ & $\begin{array}{l}\text { Fe, charitat / y } \\
\text { esperança'ns hi porten }\end{array}$ \\
\hline 421 & & & TORNADA & TORNADA \\
\hline & & $\begin{array}{l}\text { Mare de Deu / aduocada } \\
\text { mia }\end{array}$ & $\begin{array}{l}\text { Mare de Deu / aduocada } \\
\text { mia }\end{array}$ \\
\hline & $\begin{array}{l}\text { FIN DE LAS OBRAS } \\
\text { DEL EXcelente } \\
\text { Poeta Mossen Ausias } \\
\text { March. }\end{array}$ & $\begin{array}{l}\text { FI DE LAS OBRAS DEL } \\
\text { excelent Poeta Mossen } \\
\text { Ausias March }\end{array}$ \\
\hline
\end{tabular}

A continuación indicamos algunas variantes significativas para lo cual hemos agrupado testimonios $D b, b c, b d$ y $d e$. Mientras que entre $b c$ y de encontraremos básicamente erratas, las variantes más significativas de contenido estarán entre los impresos $b d$, lo que indica que no hay una relación directa entre ellos.

En primer término observamos las variantes entre $D$ (posible original manuscrito) y la edición $b$. Es habitual encontrar en el manuscrito intervenciones del copista o de otra mano del estilo: v. 63 l'<h>om / l'hom, v. 74 d'<h>om / d'hom. Entre el manuscrito y el impreso cambian las formas gráficas: v. 114 deffensa / defensa, v.118 censuals / sensuals v. 168 leia / leja, v. 171 jnteres / interés, v. 29 faç / fas; e incluso morfológicas v. 1 quim / quin, v. 38 destrus / destruus, v. 39 quit / Qui, v. 126 feneixca / fenesca, v. 142 samprenga / se prenga, v. 416 fruirem / fruireu, v. 224 los mals que si assenten / los mals que desassenten.

Algunas formas que en D aparecen interlineadas ${ }^{39}$ se reproducen integradas en el impreso: v. $418<$ la $>$ gloria / la gloria, v. 62 es com $<$ son $>$ cruel damnatge / es son cruel damnatge, o bien se tienen en cuenta las correcciones v. 420 espera / spera.

Por otra parte hay erratas evidentes tanto de $b$ : v. 24 lo sensible / la sensible, v. 381 forçat / foarçat, v. 403 grosser / gresser, como de $D$ que después corrige $b$ : v. 137 tetasta / se tasta, v. 141 sens / sent, v. 157 tot ordiment axella / tot ardiment exella, v. 206 fara / fora.

Las variantes más significativas aparecen en el siguiente cuadro:

\begin{tabular}{|l|l|}
\hline Manuscrito $D$ & Edición $b$ \\
\hline v. 174 ans cometra granperill sens ferea & ans contra / gran perill va sens ferea \\
\hline v. 188 epoch bels aconsola & e poch quels aconsola \\
\hline v. 194 esclaten & els maten \\
\hline
\end{tabular}

\footnotetext{
${ }^{39}$ No sabemos si las manipulaciones en el ms. D, como las grafías interlineadas o las correcciones al texto obedecen a los impresores, para después reproducirlo en la edición o a un corrector ajeno.
} 


\begin{tabular}{|l|l|}
\hline v. 231 Qui sols delit / odel mon te sperança & Qui vol delit / o del mon sperança \\
\hline v. 333 efora de si mora & e fora de si, ignora \\
\hline v. 352 deserta & certa \\
\hline
\end{tabular}

La edición $c$ de 1545 asume todas las variantes diferenciadas de $b$ con respecto a $D$ y no recupera lecturas del manuscrito. Otras diferencias en los impresos $b c$, son básicamente errores tipográficos, como por ejemplo: v. 3 vidam / vldam, v. 35 morint hom, tu anulles / morint hon ${ }^{40}$ tu anulles, v. 42 l'home dolre / l'hon dolre, v. 50 al hom / al ham, v. 194 els maten / ells maten, v. 216 menys de esguart ofen ${ }^{41}$ natura bona / (196) menys del'sguart o feu natura bona, v. 291 mort atraça / (271) mort atraço.

Observamos algunas variantes ortográficas v. 92 segle / setgle, v. 282 no traballen / (262) no treballen; así como erratas evidentes en $c$ : v. 123 trist / rrist, v. 160 subjugada / subjugad, v. 163 dolor / dolr, v. 232 prestey / (212) $)^{42}$ prstey, v. 243 nom de brut / (223) nom dc brut, v. 336 d'accident o natura / (316) d'accinent o natura, v. 404 qu'apres mort resta / (384) qu'apres mort rista. En algunos casos $c$ corrige unas pocas erratas que aparecían en $b$ : v. 381 foarçat es que mal vulla / (361) forçat es que mal vuulla; pero en otros casos no: v. 403 pus gresser e maligne / (383) pus gresser e maligne ${ }^{43}$.

Finalmente hay otros errores que indican un ligero cambio morfológico: v.146 desque sateny / del que sateny, v. 250 per que d'infern / (230) perque'n infern, v. 296 que no espera / (276) que no'spera, v. 303 ne desperança'ch cura / (283) ne desperança'cura, v. 384 no te loch qui aculla / (364) no te loch quel aculla, v. 416 mas fruireu / (396) mas fruirem.

Unas pocas lecturas pueden cambiar el sentido del verso:

\begin{tabular}{|l|l|}
\hline$b$ & $c$ \\
\hline v. 369 nos poden colre / & $(349)$ nos poden cloure ${ }^{44}$ \\
\hline $\begin{array}{l}\text { v. } 392 \text { quant ell a'mpres / a si lo mon } \\
\text { sots metre }\end{array}$ & $\begin{array}{l}(372) \text { quant ell a si / volgue lo mon sots } \\
\text { metre }\end{array}$ \\
\hline v. 405 via / & $(385)$ vida \\
\hline
\end{tabular}

A continuación, observamos sumariamente aquellas las variantes más significativas entre $b(1543)$ y $d(1555)$, sin tener en cuenta las tipográficas ni

\footnotetext{
${ }^{40}$ La abreviatura ha sido desarrollada como hom que es su sentido correcto. Hon es una errata de $c$.

${ }^{41}$ Posible confusión grafias $n / u$.

${ }^{42}$ Entre paréntesis los números de versos en $c$, debido a la pérdida de dos estrofas.

${ }^{43}$ Misma errata en las dos ediciones.

${ }^{44}$ Parece únicamente un error de esta edición, solo se repite en su copia manuscrita $C$ y no tiene tradición en el resto de testimonios.
} 
las ortográficas. Encontramos solo algunos casos con un ligero cambio en la morfología, por ejemplo: v. 1 quin / quim, v. 78 amants / amats, v. 128 no li falras / nol fallirlas, v. 416 fuireu (c fuirem) / fruirem, v. 231 del mon sperança / del mon te'sperança. Hay algunas expresiones que pueden cambiar el sentido del verso o bien se ha preferido utilizar sinónimos:

\begin{tabular}{|l|l|}
\hline$b$ & $d$ \\
\hline v. 21 quant est abominable & quant es a tots orrible \\
\hline v. 159 auilada & auisada (c auisada) \\
\hline v. 163 tot quant que mor & tot hom que mor \\
\hline v. 194 els maten & esclaten \\
\hline v. 195 lur / fillan mans daltri & llur fi / en mans daltri \\
\hline v. 333 ignora & $(393)^{45}$ mora \\
\hline v. 352 li esta certa & $(292)$ esta deserta \\
\hline v. 364 que toqueen descreure & $(304)$ que toch en Deu descreure \\
\hline v. 367 perdent no senfastija & $(307)$ per Deu no sen fastija \\
\hline $\begin{array}{l}\text { v. } 392 \text { quant ell ampres a si lo mon sots } \\
\text { metre }\end{array}$ & $\begin{array}{l}(332) \text { quant ell asi / volgue lo mon sots } \\
\text { metre }\end{array}$ \\
\hline v. 405 via & vida \\
\hline & \\
\hline
\end{tabular}

Por último observamos en los impresos de un tratamiento similar a los cambios de grafías, corrección de erratas o introducción de nuevos errores que caracterizan las fuentes impresas. También se observan elisiones, simplificación de consonantes dobles y, en definitiva, cambios en el nivel ortográfico que no alteran el sentido del texto, por ejemplo: v. 5 çinch / cinch, v. 14 hunida / vnida, v. 20 m'es dolentia / me es dolencia, v. 24 raçional / raonal, v. 34 cosole / console, v. 61 conexença / coneixença, v. 202 fortalea / fortalesa, v. 203 perea / peresa, v. 237 cos / cors, v. 291 Puis / Puix, v. 292 porçio / porcio, v. 339 llançes / lances, v. 389 Hagueren / Hagren. Vuelve a aparecer la confusión en el v. 405 vida / via. En cuanto a correcciones de erratas, señalamos algunas que parecen evidentes: v. 135 roho / raho, v. 286 predre / pendre, v. 321 forcat / forçat, v. 285 per fi / per si, v. 328 planga / plaga, v. 340 le diues / les diuines, v. 404 rrista / trista.

En el análisis de estas variantes recogidas en los cuatro impresos, destacaremos las siguientes:

1. Observamos cuatro casos en los que $d$ (1555) recupera la lectura del manuscrito $D$ que difiere en $b(1543)$ :

\footnotetext{
${ }^{45}$ Entre paréntesis los números de versos en $d$, debido a la no correlación entre estrofas.
} 


\begin{tabular}{|l|l|l|}
\hline$D$ & $b$ & $d$ \\
\hline v. 194 esclaten & els maten & esclaten \\
\hline $\begin{array}{l}\text { v. 231 Qui sols delit / odel } \\
\text { mon te sperança }\end{array}$ & $\begin{array}{l}\text { Qui vol delit / o del mon } \\
\text { sperança }\end{array}$ & del mon te'sperança \\
\hline v. 333 efora de si mora & e fora de si, ignora & $(393)$ mora $^{46}$ \\
\hline v. 352 deserta & li esta certa & $(292)$ esta deserta $^{47}$ \\
\hline v. 364 en deu descreure & que toqueen descreure & $\begin{array}{l}\text { (304) que toch en Deu } \\
\text { descreure }\end{array}$ \\
\hline
\end{tabular}

2. Una de las pocas lecturas en que difieren $b$ y $c$ es asumida por $d e$. La lectura de este verso que aparece en $b$ (y luego recupera $d e$ ) es la habitual en la tradición manuscrita.

\begin{tabular}{|l|l|l|}
\hline$b$ & $c / C$ & $d e$ \\
\hline $\begin{array}{l}\text { v. 392 quant ell a'mpres / } \\
\text { a si lo mon sots metre }\end{array}$ & $\begin{array}{l}\text { (372) quant ell a si / vol- } \\
\text { gue lo mon sots metre }\end{array}$ & $\begin{array}{l}\text { (332) quant ell asi / vol- } \\
\text { gue lo mon sots metre }\end{array}$ \\
\hline
\end{tabular}

$B$ : Quant ell ampres assi lomon sots metre

$D$ : Quant ell ampres assi lomon sots metre

$E$ : quant ell emprench / asi lo mon sotsmetre

$G^{3}$ : quant elanpres / assi lo mon sosmetre

3. Sin embargo, en otro caso en que los ms. $B D E G^{3}$ leemos via, $c$ interpreta vida y $d$ mantiene esta lectura, sin embargo e corrige y recupera via. El sentido de la secuencia poéticarequiere el términovia: etantesl'hompus grosseremaligne/ com no conquer lo mon qu'apres mort resta, / lo qual es fi, e lo present es via (vv. 403-405).

\begin{tabular}{|c|c|c|c|}
\hline$b$ & $c / C$ & $d$ & $e$ \\
\hline v. 405 via & vida & vida & via \\
\hline
\end{tabular}

4. En el verso 21 abominable en $D b c$ passa a orrible en de, una lectura única que solo aparece en $E$ (1546), manuscrito posterior a $b c \mathrm{y}$, como bien se argumenta en el prólogo, el texto se copia teniendo en cuenta y corrigiendo estas ediciones. Tampoco tiene tradición esta lectura, ya que los otros dos testimonios manuscritos, o bien mantienen abomjnabble $\left(G^{3}\right)$ o es una lectura diferente: noible $(H)$.

${ }^{46} B D E G^{3} H$ mora.

${ }^{47} B D G^{3} H$ deserta. E ja es cert.

${ }^{48} B G^{3}$ en deu descreure. E: en lo descreure. No en $H$. 
5. En cambio en el verso 174, contra es un posible error de $b$ que pasa al resto de impresos, también al manuscrito $E$, mientras que $G^{3}$ da otra lectura y $H$ coincide con $B D$ :

$D:$ ans cometra granperill sens ferea

$b d e:$ ans contra / gran perill va sens ferea ${ }^{49}$

$B$ : Ans cometra gran perill sens ferea

$E$ : ans contra gran / perill va sens secretari

$G^{3}$ : ans remetra / gran perill sens ferea

$H$ : Ans cometra gran perill sens peresa

Para concluir esta aproximación textual de la poesía Cobrir no pusch la dolor qui $m$ turmenta, es evidente que por su pertenencia al grupo de poesías morales escrita en estrofas de 10 versos y de extensión considerable, su transmisión es tardía en relación con el resto de poesías amorosas. En uno de los Cancioneros considerados más antiguos de March, el manuscrito $H$, se presenta en una sección diferente, incompleta y con bastantes posibilidades que la copia sea posterior y de una mano diferente a la del resto del manuscrito. Por otra parte, este testimonio contiene dos secciones más de la poesía marquiana y en total 79 composiciones. Ocurre lo mismo con el manuscrito $G$, la poesía ocupa una única sección $G^{3}$ y no parece tener relación con el testimonio $H$.

El grupo de manuscritos $B D$ y los impresos $b c$, realizados en torno a la figura de Ferrando Folch de Cardona en la primera mitad del siglo XVI, consolida el texto de la extensa composición, en el cual encontramos pocas variantes de sentido. A partir de estos testimonios, con algunas omisiones en la edición de 1545 , la composición aparece regularmente y con un texto estable. Se observa en el primer impreso de 1543 la dependencia del manuscrito $D$ que posiblemente sirvió de original así como una serie de erratas en $c$ (1545) que hacen pensar en un mayor descuido de los impresores a la hora de elaborar la reedición, incluso la desaparición de dos estrofas de la poesía 112.

Aunque los manuscritos $B$ y $D$ no presentan la división en secciones, las primeras ediciones renacentistas someten el conjunto poético a una reordenación temática en la que la composición 112 ocupará la sección de Obras o Cantos Morales por su temática de reflexión sobre la muerte y los bienes desaparecidos a consecuencia de ésta. Por lo que respecta a las ediciones más recientes de 1555 y 1560 (de), la lírica del poeta valenciano se somete a

\footnotetext{
${ }^{49}$ Este verso suprimido en $c C$.
} 
un nuevo proceso de reorganización y se incluye esta composición entre las poesías de muerte.

La regularización a que se sometió el texto en los procesos de impresión y su dependencia de una tradición manuscrita tardía son los posibles factores por los que no encontramos una lecturas sumamente diferenciadas, aunque sí diversidad de erratas, cambios de grafías que incluso se advierte una evolución en la ortografía. Aunque no tenemos en cuenta las variantes de los manuscritos, sí que observamos alguna lectura única en $H$, testimonio que parece no influyó en los impresos como tampoco $G^{3}$. La estabilidad del texto es lo que predomina en los impresos con las escasas variantes entre el posible original manuscrito $D$ y la edición de 1543 , unas variantes que, en algunos casos recupera la edición $d$.

Encontramos también algún caso de interferencias en las ediciones más tardías (de) con el manuscrito $E$, de 1546. En el prólogo de este testimonio se alude a que el copista pretende corregir algunas lecturas de las poesías que aparecen en las ediciones de 1543 y 1545, además se introdujeron lecturas diferentes, posiblemente con ánimo de mejorar la interpretación de las poesías, así como también una reordenación alfabética de las composiciones que no prosperó en las ediciones posteriores ni tampoco representa un deseo de ordenación temática.

Finalmente, cabe destacar la transmisión tardía de la poesía 112 por su inclusión en una sección única en $H G^{3}$ y porque es a partir de 1541, con las copias patrocinadas por Ferrando Folch de Cardona, cuando la encontramos formando parte del Cancionero marquiano. La composición 112 se incluye entre las composiciones morales, considerándose la totalidad de la obra de Ausiàs como un devenir cronológico: amor, muerte y reflexión moral o espiritual. Sin embargo por el tema tratado, las dos últimas ediciones del siglo XVI incluyen la poesía 112 entre los Cants de Mort, poesías con las que no tiene relación textual ni temática.

Recibido: 3/03/2020

Aceptado: 18/04/2020 


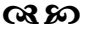

«COBRIR NO PUSCH LA DOLOR QUI·M TURMENTA»: FUENTES IMPRESAS Y TRADICIÓN MANUSCRITA DE UNA POESÍA DE AUSIÀS MARCH SOBRE LA MUERTE

RESUMEN: La tradición textual de la poesía 112 de Ausiàs March, una de las más extensas de su Cancionero, presenta algunas peculiaridades. Copiada en el ms. H, uno de los más antiguos por una mano posterior, se populariza su difusión dentro de la sección «cantos morales» con los manuscritos de autor y las ediciones impresas del siglo XVI. El estudio de los impresos revela la relación de dependencia entre las dos ediciones barcelonesas de 1543 y 1545, así como entre las ediciones de 1555 (Valladolid) y 1560 (Barcelona). Por su transmisión posiblemente tardía, la poesía no presenta unas variantes de consideración, aunque algunas lecturas remiten a la tradición manuscrita anterior. También se observan interferencias con el texto copiado en 1546 , el manuscrito $\mathrm{E}$, fechado con posterioridad a las primeras ediciones de la obra del poeta valenciano.

Palabras Clave: Ausiàs March. Poesia 112. Tradición textual. Variantes impresos. Muerte.

\footnotetext{
«COBRIR NO PUSCH LA DOLOR QUI•M TURMENTA»: PRINTED SOURCES AND HANDWRITTEN TRADITION OF AN AUSIÀS MARCH POEM ABOUT DEATH
}

ABSTRACT: The textual tradition of poetry 112 by Ausiàs March, one of the most extensive of its Cancionero, presents some peculiarities. It was copied in $\mathrm{H}$, one of the oldest manuscripts by a later hand, but its diffusion was popularized within the section «moral songs» in the manuscripts of author and the printed editions of the XVI century. The study of the printed books reveals the relationship of dependence between the two Barcelona editions of 1543 and 1545, as well as between the editions of 1555 (Valladolid) and 1560 (Barcelona). Because of its possibly late transmission, poetry does not present some variants of consideration although some readings refer to the previous manuscript tradition. Interferences are also observed with the text copied in 1546, the manuscript $\mathrm{E}$, dated after the first editions of the work of the Valencian poet.

KeYwords: Ausiàs March. Poetry 112. Textual tradition. Printed variants. Death. 